\title{
Beam-Beam Interactions at the Tevatron in Run IIa
}

\author{
T. Sen, N. Gelfand and M. Xiao \\ FNAL, P.O. Box 500, Batavia, IL 60510
}

\begin{abstract}
The Tevatron in Run IIa will operate with three trains of twelve bunches each. The impact of the long-range interactions on beam stability will be more significant compared to Run I. We study these beam-beam interactions (head-on and long-range) with particle tracking using two different codes. The model includes machine nonlinearities such as the field errors of the Interaction Region quadrupoles and the chromaticity sextupoles. Tune footprints and dynamic apertures are calculated for different bunches in a train.
\end{abstract}

\section{INTRODUCTION}

The Tevatron will operate again in collider mode starting in summer 2001. In the first phase, termed Run IIa, $36 \bar{p}$ bunches in three trains of twelve bunches will collide with 36 proton bunches. This is a six-fold increase in the number of bunches from the last collider operation Run Ib. Design proton intensities are higher so the head-on beambeam tune shifts experienced by the anti-protons will be higher than in Run Ib. The greater number of long-range beam-beam interactions will increase the total beam-beam induced tune spread of the anti-protons. Furthermore these effects are different for each $\bar{p}$ bunch in a train since the sequence of long-range interactions is different for each of them. All of these effects taken together may reduce the dynamic aperture (DA) and/or lifetime of the anti-protons significantly. In this report we calculate the DA of a few bunches including the nonlinear fields in the IR quadrupoles amongst the nonlinearities. In the second stage of Run II, the plan is to increase the luminosity further with more intense bunches, larger number of bunches to decrease the number of interactions per bunch crossing, and also introduce crossing angles at B0 and D0 to avoid parasitic collisions with zero separation.

\section{BEAM-BEAM INTERACTIONS IN RUN IIA}

The design luminosity will increase from Run I to Run II mainly with an increase in the proton intensities and the number of bunches. Table 1 shows the main beam parameters. In Run IIa each bunch will experience two head-on interactions at B0 and D0 and seventy long-range interactions. These long-range interactions will be distributed over the entire ring with differing beam separations and differing phase advances from one interaction to the next. This sequence of interations will also be different for every bunch in the train, e.g. the leading bunch 1 will experience all long-range interactions downstream of the IP, bunch 6 will experience five interactions upstream and six interactions
Table 1: Main beam parameters in Run I and Run II

\begin{tabular}{|l|l|l|}
\hline & $\begin{array}{l}\text { Run Ib } \\
p / \bar{p}\end{array}$ & $\begin{array}{l}\text { Run IIa } \\
p / \bar{p}\end{array}$ \\
\hline Luminosity $\left[\mathrm{cm}^{-2} \mathrm{sec}^{-1}\right.$ ] & $1.6 \times 10^{31}$ & $8.6 \times 10^{31}$ \\
Bunch Intensities $\times 10^{11}$ & $(2.3 / 0.55)$ & $(2.7 / 0.3)$ \\
Emittances 95\% [mm-mrad] & $23 / 13$ & $20 / 15$ \\
Number of bunches & 6 & 36 \\
Bunch separation [m] & 1049.3 & 118.8 \\
Beam size at IP $[\mu \mathrm{m}]$ & $37 / 28$ & $33 / 29$ \\
Beam-beam parameter/IP $\times 10^{-3}$ & $3.4 / 7.4$ & $1.5 / 9.9$ \\
\hline
\end{tabular}

downstream of the IP etc. The tune footprint will therefore differ from bunch to bunch. The nominal working point $\left(\nu_{x}=0.585, \nu_{y}=0.575\right)$ is chosen to lie between fifth and seventh order resonances. At this working point the Tevaton beam straddles twelfth order sum resonances. Operational experience during Run I showed that these resonances did not cause a significant reduction in lifetime. However the tune footprints and nonlinearities were also smaller in Run I. Figure 4 shows the footprints due to the beam-beam interactions in Run IIa for bunch 1 and bunch 6 superposed on nearby sum resonances up to twelfth order. Footprints of all bunches except for bunch 1 and 12 are clustered around that of bunch 6 . The major differences in the tuneshifts between bunch 6 and bunch 1 and 12 are due to the missing parasitic collision closest to the IP, upstream for bunch 1 and dowstream for bunch 12 . The variation in the tune shift and in the tune spread from bunch to bunch will be greatly enhanced in Run IIb when the number of bunches is increased to more than one hundred. The Tevatron beam-beam compensation project [1] aims to reduce this spread in tunes by colliding anti-proton bunches with a low energy electron beam whose intensity will be varied from bunch to bunch. However even in Run IIa, the stronger beam-beam interactions at the IP $(\xi \approx 0.01$ compared to $\xi \approx 0.074$ in Run Ib) and the larger number of long-range interactions may cause emittance growth and reduced lifetime of the anti-protons.

Figure 1 shows the beam separation (in units of the rms bunch size) at all the seventy two locations of beam-beam interactions for bunch 6. At most locations the beam separation is of the order of $10 \sigma$. The prominent exceptions are the parasitic collisions nearest to the IPs where the separation is only about $6 \sigma$. These nearest interactions in fact also have the dominant contribution to the tune footprint. 


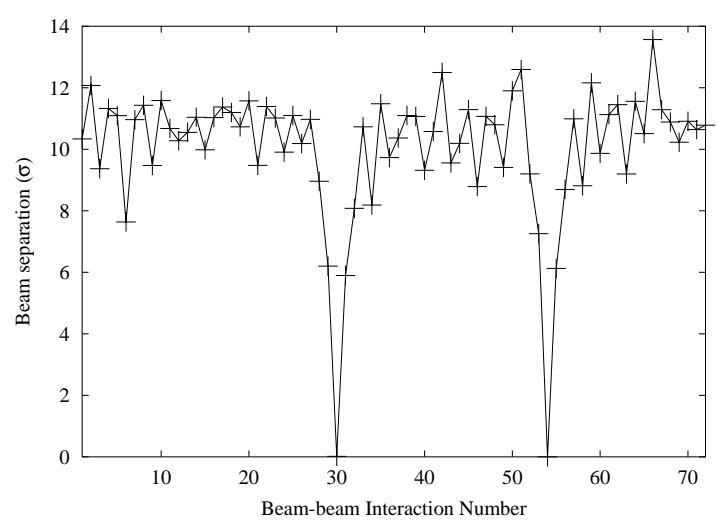

Figure 1: Separation between $\bar{p}$ bunch 6 and the opposing proton bunch at all 72 beam-beam interactions. The headon collisions are at locations 30 and 54 .

\section{FOOTPRINTS AND DYNAMIC APERTURE CALCULATIONS}

The bunch length in the Tevatron is comparable to the beta function at the IP, $\sigma_{s} \approx 37 \mathrm{~cm}, \beta^{*}=35 \mathrm{~cm}$. Recent theoretical work [2] has shown that bunch length effects which include hour glass effects and phase averaging reduce resonance strengths in the Tevatron by two orders of magnitude compared to strengths calculated with zero length bunches. We include these bunch length effects in the simulations of the head-on interactions described below. The long-range effects are modelled by delta function kicks.

The simulation model includes the beam-beam kicks, the nonlinear fields in the Interaction Region (IR) quadrupoles (the beta functions in these magnets is about three to four times the values in the arcs) and the chromaticity correcting sextupoles. Two simulation programs MAD and TEVLAT are used to track particles. In most cases the DA calculated by these codes are within $2 \sigma$ (or about $15 \%$ ) of each other. In this report linear imperfections such as orbit errors and coupling due to misalignments are not included and neither are time-dependent effects such as those due to power supply ripple. Synchrotron oscillations and other momentum dependent effects have yet to be studied in sufficient detail. These effects are important and will be included in further studies.

Figure 2 shows the tune footprint for $\bar{p}$ bunch 6 in two cases. The addition of the long-range interactions increases the tune spread significantly and particles at amplitudes up to approximately $3 \sigma$ now straddle the 5 th and 10th order resonances. Inclusion of the IR errors does not change the footprint significantly.

Tracking to calculate the DA was done for $10^{5}$ and $10^{6}$ turns. For $10^{5}$ turns, particles were launched at several transverse amplitude ratios. Both the average and the minimum over these values are taken as measures of the DA. Figure 3 shows the DA of bunch 6 with only the beam-beam interactions in one case and with all the beam-beam kicks and machine nonlinearities in the other case. For compari-

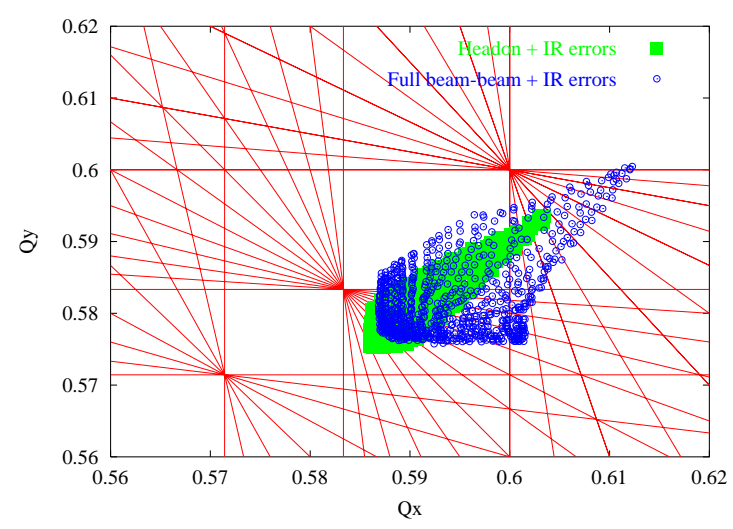

Figure 2: (color) Tune fooprint of $\bar{p}$ bunch 6 with (i)the head-on interactions and (ii) all the beam-beam interactions. IR errors and chromaticity sextupoles are included in each case. The footprint is shown for particles with amplitudes up to $6 \sigma$. Nearby fifth, seventh, tenth and twelfth order sum resonances are shown. The linear lattice tune is $(0.585,0.575)$.

son, the DA with only head-on and IR errors is also shown. In all cases the chromaticity sextupoles are included. It is evident that the long-range interactions cause a sharp drop in the DA. For example, the minimum DA drops from $13.6 \sigma$ to $8.6 \sigma$ when these interactions are included (see Table 2). The IR errors have a smaller relative impact, they reduce the average DA by about $1.6 \sigma$. The relative importance of these IR errors could change when crossing angles are introduced and particle orbits go significantly off-axis through these magnets.

We have also calculated the DA when the only longrange interactions are the nearest parasitic collisions (PCs) to the IPs. We find that with only these PCs both the average and minimum DA are about $2 \sigma$ smaller but not quite as small as when all the PCs are included, see Table 2. It is therefore not obvious that reducing the tune spread alone, for example by choosing a different bunch spacing so that only the nearest PCs are further apart, would necessarily improve the DA.

The DA is sensitive to the machine tune. One might choose the tunes so that only small amplitude particles $(<$ $1 \sigma$ ) straddle the fifth and tenth order resonances. Calculations with a few tunes chosen to accomplish this do not appear to improve the DA significantly, see Table 2.

We find that when the long-range interactions are added, tune diffusion increases by an order of magnitude in regions close to the diagonal $(x=y)$ in amplitude space but is relatively unchanged along the $x$ and $y$ axes. Figure 3 shows that the DA drops the most close to the diagonal when the long-range interactions are added.

Longer term tracking ( $10^{6}$ turns) was done only along the diagonal in amplitude space for bunches 1, 6 and 12. Figure 5 shows the survival plot - the number of turns survived as a function of the initial amplitude - for these bunches. Bunch 1 appears to be the least stable and bunch 12 the most sta- 


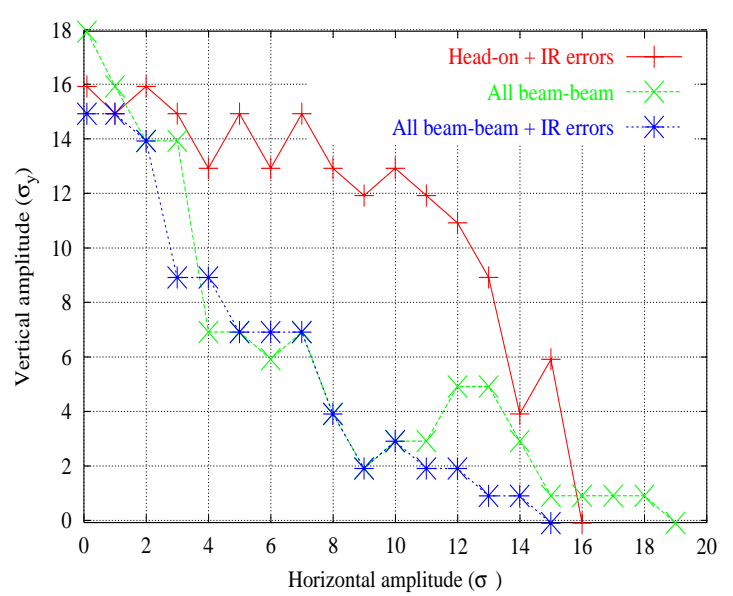

Figure 3: (color) DA for bunch 6 with (i) all beam-beam kicks and (ii) all beam-beam and the IR errors. For comparison, the DA with only head-on interactions and IR errors is also shown.

Table 2: DA in $\sigma$ for bunches 6,1 and 12

\begin{tabular}{|l|l|l|}
\hline & $\langle D A\rangle$ & $D A_{\min }$ \\
\hline \multicolumn{3}{|c|}{ Bunch 6: $\nu_{x}=0.585, \nu_{y}=0.575$} \\
DA after 105 turns \\
IR errors & 18.7 & 17.0 \\
Head-on + IR errors & 15.5 & 13.6 \\
Head-on,nearest PCs, IR errors & 13.6 & 11.3 \\
All beam-beam & 12.9 & 8.1 \\
All beam-beam + IR errors & 11.3 & 8.6 \\
\hline \multicolumn{3}{|c|}{ All beam-beam + IR errors } \\
$\nu_{x}=0.572, \nu_{y}=0.574$ & 11.0 & 10.0 \\
$\nu_{x}=0.591, \nu_{y}=0.580$ & 8.5 & 7.6 \\
$\nu_{x}=0.575, \nu_{y}=0.585$ & 9.5 & 8.3 \\
\hline \multicolumn{3}{|c|}{ DA after 106 turns } \\
Bunch 1 & 6.4 \\
Bunch 6 & 7.8 \\
Bunch 12 & 8.5 \\
\hline
\end{tabular}

ble. The maximum variation in DA from bunch to bunch is $2 \sigma$. Even after $10^{6}$ turns there is no indication that the stable amplitude is levelling off for either of these bunches. Since $10^{6}$ turns corresponds to about 21 seconds in the Tevatron, particles at smaller amplitudes than seen in Figure 5 and in Table 2 could be lost over a time scale of minutes. During operation it is only necessary that the real DA exceed the physical aperture which is defined by the primary collimators which are at $\simeq 6 \sigma$ from the beam core. Given that many effects mentioned above were not included in the simulation model, the real DA of nearly all bunches is likely to be smaller than the physical aperture.

Conditions in Run IIb will be significantly more challenging. One new aspect will be the crossing angles at B0 and D0. Studies have shown [2] that at well chosen tunes, the synchro-betatron resonances driven by the beam-

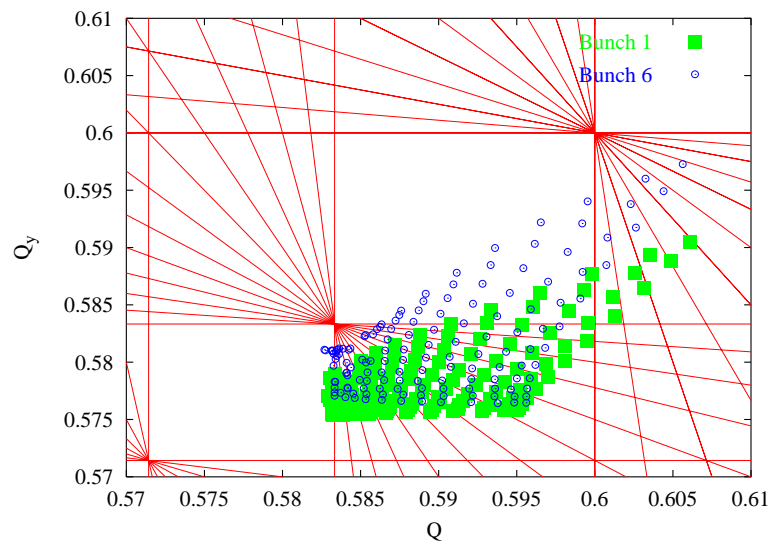

Figure 4: (color) Tune footprint for bunches 1 and 6 in a train.

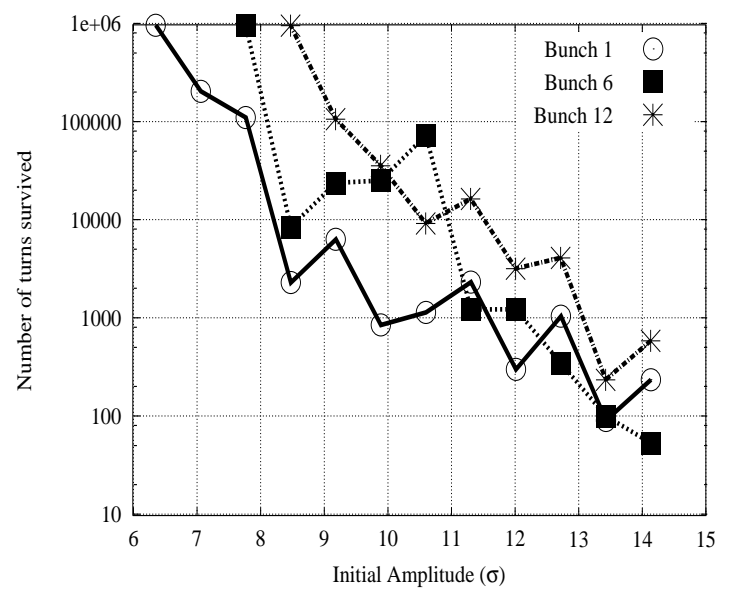

Figure 5: Survival plot for bunches 1,6 and 12 up to $10^{6}$ turns. All beam-beam kicks and machine nonlinearities are included.

beam interactions do not by themselves cause significant amplitude growth up to crossing angles of $500 \mu$ radians the maximum thought necessary. The choice of optimum crossing angle however will be determined by considering all the nonlinearities - a study that is in progress.

\section{CONCLUSIONS}

Calculations assuming ideal machine conditions show that the dynamic aperture of most bunches in Run IIa is smaller than $8 \sigma$. In most realistic operating conditions the dynamic aperture will likely be smaller than the physical aperture of $6 \sigma$. The stable boundary in Run IIb will be significantly smaller unless the long-range interactions are compensated.

\section{REFERENCES}

[1] V. Shiltsev, this conference

[2] T. Sen, Fermilab preprint, to be published 